(6) OPEN ACCESS

\title{
Persistence after treatment of pharyngeal gonococcal infections in patients of the STI clinic, Amsterdam, the Netherlands, 2012-2015: a retrospective cohort study
}

\author{
I Putu Yuda Hananta, 1,2 Henry John Christiaan De Vries, ${ }^{1,2}$ Alje P van Dam, ${ }^{2,3}$ \\ Martijn Sebastiaan van Rooijen, ${ }^{2}$ Hardyanto Soebono, \\ Maarten Franciscus Schim van der Loeff ${ }^{2,5}$
}

'Department of Dermatology, Academic Medical Center University of Amsterdam, Amsterdam, The Netherlands ${ }^{2}$ Department of Infectious Diseases, Public Health Service (GGD), Amsterdam, The Netherlands

${ }^{3}$ Department of Medical Microbiology, Onze Lieve Vrouwe Gasthuis (OLVG) Hospital, Amsterdam, Netherlands

${ }^{4}$ Department of Dermatology and Venereology, Faculty of Medicine, Universitas Gadjah Mada, Yogyakarta, Indonesia ${ }^{5}$ Department of Internal Medicine, Center for Infection and Immunology Amsterdam (CINIMA), Academic Medical Center, University of Amsterdam Amsterdam, The Netherlands

\section{Correspondence to}

Dr Maarten Franciscus Schim van der Loeff, Department of Infectious Diseases, Public Health Service (GGD) of Amsterdam, Nieuwe Achtergracht 100, 1018 WT Amsterdam, The Netherlands; mschimvdloeff@ggd. amsterdam.nl

Received 9 February 2017 Revised 20 April 2017 Accepted 10 June 2017 Published Online First 19 August 2017

\section{SLinked}

- http://dx.doi.org/10.1136/ sextrans-2016-052950

\section{CrossMark}

\footnotetext{
To cite: Hananta IPY, De Vries HJC, van Dam AP, et al. Sex Transm Infect 2017:93:467-471.
}

\begin{abstract}
Introduction Infection of Neisseria gonorrhoeae in the pharynx (pharyngeal $\mathrm{Ng}$ ) is associated with gonococcal transmission and development of antimicrobial resistance. We assessed proportion of and determinants for persistence after treatment of pharyngeal $\mathrm{Ng}$.

Methods At the STI clinic of Amsterdam, the
\end{abstract} Netherlands, females-at-risk and men who have sex with men are routinely screened for pharyngeal $\mathrm{Ng}$ using an RNA-based nucleic acid amplification test (NAAT; Aptima Combo 2). Patients with pharyngeal $\mathrm{Ng}$ were invited for a test-of-cure (TOC) 7 days after treatment with a $500 \mathrm{mg}$ ceftriaxone intramuscularly. We retrospectively examined medical records of patients with pharyngeal $\mathrm{Ng}$ (January 2012-August 2015) who returned for a TOC 7-28 days after treatment. Persistence was defined as a positive NAAT at TOC.

Results Out of 2204 pharyngeal $\mathrm{Ng}$ cases recorded in the study period, 781 cases (median time between first treatment and TOC of 8 (IQR 7-12) days) were included in the analysis. Persistence after treatment was found in $36(4.6 \%)$ and was less likely among patients who received ceftriaxone in combination with other antibiotics (vs monotherapy) (adjusted OR (aOR) 0.36, 95\% Cl 0.12 to 1.04) and with longer time from treatment to TOC (aOR $0.74,95 \%$ Cl 0.60 to 0.90 , per extra day). In those with a TOC 15-28 days after treatment, $\mathrm{Ng}$ persisted in only $1.0 \%$ ( $1 / 105$ cases).

Conclusion A small proportion of pharyngeal $\mathrm{Ng}$ persists despite appropriate treatment. Combining ceftriaxone with other antibiotics appears to lead to faster clearance. A TOC for pharyngeal Ng 7 days after treatment may be too soon.

\section{INTRODUCTION}

Pharyngeal infection by Neisseria gonorrhoeae (pharyngeal $\mathrm{Ng}$ ) accounts for up to $16.5 \%$ of all gonorrhoea cases, especially in men who have sex with men (MSM). ${ }^{1-5}$ Through various types of sexual contact involving oropharyngeal mucosa and saliva, pharyngeal $\mathrm{Ng}$ may generally sustain gonococcal transmission in the society. ${ }^{126}$

Extended spectrum cephalosporins (ESCs), for example, ceftriaxone or cefixime, are currently the standard treatment for gonorrhoea, including pharyngeal Ng. ${ }^{2}$ However, persisting pharyngeal $\mathrm{Ng}$ despite appropriate antibiotic treatment has been reported, ${ }^{478}$ and such persistence was associated with antimicrobial resistance against ESCs. ${ }^{4}$

We aimed to assess determinants of persistence after treatment of pharyngeal $\mathrm{Ng}$, which could be useful for appropriate management of pharyngeal $\mathrm{Ng}$ in clinical practice.

\section{METHODS}

Routine pharyngeal $\mathrm{Ng}$ management at the study setting

At the STI clinic, Public Health Service, Amsterdam, the Netherlands, females-at-risk (ie, those who reported receptive oral sex in the preceding 6 months, had been notified of an STI or received money for sex) and all MSM are routinely screened for pharyngeal $\mathrm{Ng}$ using an RNA-based nucleic acid amplification test (NAAT), the Aptima Combo 2 Assay (Gen-Probe Diagnostics, San Diego, California, USA). We defined the day when the patients were screened for pharyngeal $\mathrm{Ng}$ as 'the first consultation'.

Patients are requested to return for treatment on a positive NAAT result. However, an immediate treatment might be administered at the first consultation if patient reported genital, anorectal or pharyngeal discharge or pain and had a Gram stained smear of an anogenital sample containing intracellular Gram negative diplococci on light microscopical examination or had been notified for gonorrhoea. The standard treatment for pharyngeal $\mathrm{Ng}$ is a single dose of ceftriaxone $500 \mathrm{mg}$ intramuscular. An alternative regimen might be prescribed due to a history of allergy or in case of coinfections requiring additional antibiotics. Depending on the clinical response, some patients might receive several treatment courses for one episode of infection.

Prior to treatment, pharyngeal swabs are also collected for culture and antibiotic susceptibility test (AST) against ceftriaxone, cefixime, cefotaxime, azithromycin, doxycycline and ciprofloxacin, using the Etest method (BioMerieux, Marcy l'Etoil, France), as described (http://www.biomerieux-usa.com/clinical/etest).

All patients with pharyngeal $\mathrm{Ng}$ were invited for a test-of-cure (TOC) 7 days after treatment. 


\section{Definitions and inclusion/exclusion criteria}

We retrospectively examined a cohort of patients diagnosed with pharyngeal $\mathrm{Ng}$ at the STI clinic Amsterdam between 1 January 2012 and 31 August 2015, using electronic medical records data. Demographics and behaviour data were collected using a self-administered questionnaire.

Inclusion criteria for the study were: (1) receiving ceftriaxone as the first treatment (as monotherapy or in combination with antibiotics that are potentially effective to treat pharyngeal $\mathrm{Ng}$, ie, azithromycin, doxycycline, amoxicillin, ciprofloxacin, ofloxacin or benzylpenicillin) and (2) returning for TOC 7-28 days after treatment.

Exclusion criteria were: (1) receiving additional antibiotics between the first treatment and TOC and (2) spontaneous clearance, that is, a negative NAAT result prior to treatment (if cases were not treated immediately).

Persistence was defined as a positive NAAT result at the first TOC visit 7-28 days after treatment (TOC 1 ). If cases persisted at TOC $_{1}$, we followed until 28 days after treatment and reported additional treatments and NAAT results (referred to as $\mathrm{TOC}_{2}$ ).

We also examined culture and AST results against ceftriaxone of isolates collected prior to treatment.

\section{Statistical analysis}

Statistical analysis was performed in STATA V.13 (StataCorp, College Station, Texas, USA). We compared characteristics of included and excluded patients using $\chi^{2}$ test for categorical variables and Kruskal-Wallis test for continuous variables. $\mathrm{p}<0.05$ was considered significant.

Bivariable and multivariable analyses for determinants of persistence after treatment were performed using logistic regression; the ORs, their 95\% CI and p values were provided. For the multivariable model, in addition to age group as an a priori variable, a more strict approach was applied by only including variables which in bivariable analysis showed $\mathrm{p} \leq 0.10$. Using a backward selection approach, the likelihood ratio test assessed the contribution of each variable to the model. We obtained a final model consisting of the a priori variable and all variables with $\mathrm{p}<0.05$.

We reported antibiogram results of cases with a successful pharyngeal $\mathrm{Ng}$ culture prior to treatment. Differences in the distribution of minimum inhibitory concentration (MIC) against ceftriaxone between cases that cleared infection and those that persisted were examined using the Wilcoxon rank-sum test.

\section{RESULTS}

Between January 2012 and August 2015, pharyngeal Ng was diagnosed in a total of 2204 consultations at the STI clinic Amsterdam: 1984 among MSM and 220 among females-atrisk. We excluded 1423 patients from the analysis because of incomplete treatment information $(n=87)$, spontaneous clearance $(n=139)$, not receiving ceftriaxone as first treatment $(n=205)$ or not returning for TOC 7-28 days after treatment $(\mathrm{n}=992)$. The remaining 781 patients were included in the analysis.

Compared with excluded patients, included patients were older (median age, IQR] of $32(25-41)$ vs 31 (25-39) years), less frequently reported genital, anorectal or pharyngeal symptoms $(28.8 \%$ vs $41.1 \%)$, were less frequently diagnosed with chlamydia or syphilis $(18.4 \%$ vs $22.5 \%)$, were less likely to have HIV (22.5\% vs 26.4\%), and reported a longer median time from first consultation to treatment (8 (3-11) vs $7(0-11)$ days).
However, the two groups did not differ in terms of gender (90.4\% male vs $89.8 \%$ ), median number of sex partners in the preceding 6 months (7 (4-15) vs $8(4-15))$, history of receptive oral sex (100\% vs $99.9 \%)$, history of sex work (6.0\% vs $6.5 \%)$, being notified for gonorrhoea $(24.0 \%$ vs $23.6 \%)$ and having $N$. gonorrhoeae coinfections at other anatomical locations $(41.6 \%$ vs $46.2 \%)$.

Median time from treatment to TOC $_{1}$ was 8 (7-12) days. Persistence after treatment was observed in $36 / 781$ cases (4.6\%). In bivariable analysis, variables that were associated with persistence at $\mathrm{p} \leq 0.10$ were history of sex work (OR 2.71, 95\% CI 1.00 to 7.35 ), combination of ceftriaxone and other antibiotics as treatment regimen (vs ceftriaxone as monotherapy, OR $0.29,95 \%$ CI 0.10 to 0.84 ) and time from treatment to $\mathrm{TOC}_{1}$ (OR $0.72,95 \%$ CI 0.59 to 0.89 , per extra day) (table 1 ).

In the multivariable model, persistence was less likely in those who were treated with combination of ceftriaxone and other antibiotics (aOR $0.36,95 \%$ CI 0.12 to 1.04 ) and in those with a longer time from treatment to $\mathrm{TOC}_{1}$ (aOR 0.74, 95\% CI 0.60 to 0.90 , per extra day) (table 1 ).

Among 36 patients with persistence at $\mathrm{TOC}_{1}, 15$ returned for $\mathrm{TOC}_{2}$ (among which four received additional ceftriaxone between $\mathrm{TOC}_{1}$ and $\mathrm{TOC}_{2}$ ), whereas 21 were lost to follow-up. At $\mathrm{TOC}_{2}$, infection was cleared in 14 and had persisted in one case.

Antibiograms of isolates prior to first treatment were available for 149/781 patients. The median and range of MIC against ceftriaxone did not differ between 128 cases that had cleared the infection at TOC $_{1}(0.004(0.002-0.125) \mathrm{mg} / \mathrm{L})$ and the 21 cases that persisted $(0.004(0.002-0.047) \mathrm{mg} / \mathrm{L})$. The case that persisted at $\mathrm{TOC}_{2}$ was susceptible to ceftriaxone (MIC of $0.023 \mathrm{mg} / \mathrm{L})$.

\section{DISCUSSION}

We reported pharyngeal gonorrhoea cases that persisted despite appropriate treatment. The proportion of persisted cases was smaller compared with previous studies. ${ }^{14578}$ Ceftriaxone monotherapy seems to remain effective to treat pharyngeal $\mathrm{Ng}$, as suggested. ${ }^{278}$ Yet, combining ceftriaxone with another antibiotic appears to lead to faster clearance.

Adequate timing that allows complete clearance with minimum chance for re-exposure is crucial in pharyngeal $\mathrm{Ng}$ TOC. The rate of clearance may be influenced by infection site, diagnostic standard and treatment regimen. ${ }^{14-10}$ We found that the time to clearance of pharyngeal Ng RNA after appropriate treatment is relatively short. ${ }^{458} \mathrm{~A}$ time-dependent effect of ceftriaxone might be explained by its pharmacodynamic properties. ${ }^{9}$ The recommended timing for pharyngeal $\mathrm{Ng}$ TOC using NAAT varies from 7 to 30 days after treatment, ${ }^{4-8}$ but our study showed that TOC 7 days after treatment may be too soon.

Antimicrobial resistance is an unlikely explanation for persistent pharyngeal $\mathrm{Ng}$ in our study: isolates of cleared and persisting cases showed a similar distribution of MIC against ceftriaxone, and none was resistant.

Our study has several limitations. Most culture for pharyngeal samples was not successful and could not be used for confirmation. The use of NAAT to detect actual active infection may not be ideal either. A highly sensitive NAAT could detect genetic materials of non-viable organisms promptly following an exposure or during infection resolution. ${ }^{1610}$ Because samples were only tested at patients' visit, the infection duration could not be precisely estimated. Information on re-exposure between the first and subsequent consultations was unavailable; therefore, 


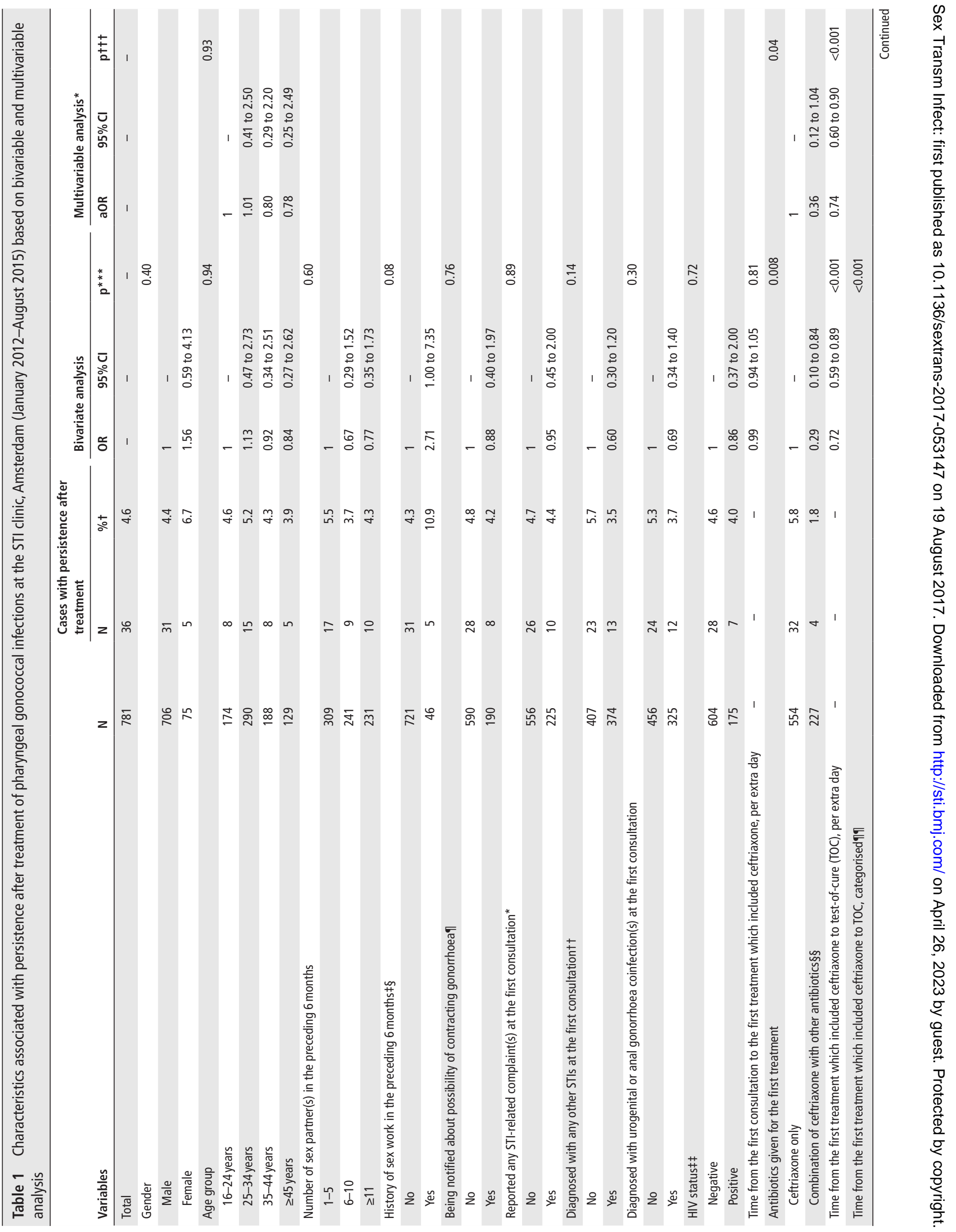


the possibility that cases of observed persistence may have been reinfections after successful treatment could not be ruled out. Finally, the small number of cases with persistence after treatment limited the power of analysis.

Our study also has several strengths. We analysed a large number of cases that were systematically recorded during a long period. The data were collected from a routine public health STI service in a large city setting.

\section{CONCLUSIONS}

A small proportion of pharyngeal $\mathrm{Ng}$ persists despite appropriate treatment. Combining ceftriaxone with other antibiotics appears to lead to faster clearance. TOC for pharyngeal $\mathrm{Ng} 7$ days after treatment may be too soon. Prospective studies are needed to produce definite evidence for the most appropriate treatment regimen for pharyngeal $\mathrm{Ng}$ and the most appropriate timing for TOC.

Handling editor Jackie Cassell

Acknowledgements We would like to express the greatest appreciation to the clinicians and laboratory technicians at the STI clinic Public Health Service (GGD) of Amsterdam.

Contributors IPYH, HJCDV, APVD and MFSVDL contributed to the design of the study. MSVR prepared the data set using clinic's electronic medical records. IPYH performed data cleaning and analyses. IPYH prepared the manuscript draft. All authors contributed to the interpretation of data and revising the manuscript critically for important intellectual content. All authors saw and approved the final version of the manuscript. All authors agreed to be accountable for all aspects of the work.

Funding This work was funded by Public Health Service (GGD) of Amsterdam, the Netherlands and the Excellence Scholarship Program (Program Beasiswa Unggulan) organised by Ministry of Research, Technology and Higher Education, Republic of Indonesia.

Competing interests HJCDV and MFSVL served on a vaccine advisory board of GSK. Other authors have no conflict of interest to declare.

Patient consent Obtained.

Ethics approval As this was a retrospective cohort study using only routinely obtained data, no ethical clearance or informed consent was required.

Provenance and peer review Not commissioned; externally peer reviewed.

Data sharing statement No additional data are available.

Open Access This is an Open Access article distributed in accordance with the Creative Commons Attribution Non Commercial (CC BY-NC 4.0) license, which permits others to distribute, remix, adapt, build upon this work non-commercially, and license their derivative works on different terms, provided the original work is properly cited and the use is non-commercial. See: http://creativecommons.org/ licenses/by-nc/4.0/

(c) Article author(s) (or their employer(s) unless otherwise stated in the text of the article) 2017. All rights reserved. No commercial use is permitted unless otherwise expressly granted.

\section{REFERENCES}

1 Chan PA, Robinette A, Montgomery M, et al. Extragenital infections caused by Chlamydia trachomatis and Neisseria gonorrhoeae: a review of the literature. Infect Dis Obstet Gynecol 2016;2016:1-17.

2 World Health Organization. WHO guideline for the treatment of Neisseria gonorrhoeae. Geneva: WHO Press, 2016.

3 European Centre for Disease Prevention and Control. Annual epidemiological report 2013, reporting on 2011 surveillance data and 2012 epidemic intelligence data. Stockholm: ECDC, 2013.

4 Bissessor M, Whiley DM, Fairley CK, et al. Persistence of Neisseria gonorrhoeae DNA following treatment for pharyngeal and rectal gonorrhea is influenced by antibiotic susceptibility and reinfection. Clin Infect Dis 2015:60:557-63.

5 Ota KV, Fisman DN, Tamari IE, et al. Incidence and treatment outcomes of pharyngeal Neisseria gonorrhoeae and Chlamydia trachomatis infections in men who have sex with men: a 13-year retrospective cohort study. Clin Infect Dis 2009;48:1237-43.

6 Chow EP, Camilleri S, Ward C, et al. Duration of gonorrhoea and chlamydia infection at the pharynx and rectum among men who have sex with men: a systematic review. Sex Health 2016;13:199-204. 
7 Barbee LA, Kerani RP, Dombrowski JC, et al. A retrospective comparative study of 2-drug oral and intramuscular cephalosporin treatment regimens for pharyngeal gonorrhea. Clin Infect Dis 2013;56:1539-45.

8 Singh AE, Gratrix J, Martin I, et al. Gonorrhea treatment failures with oral and injectable expanded spectrum cephalosporin monotherapy vs dual therapy at 4 canadian sexually transmitted Infection clinics, 2010-2013. Sex Transm Dis 2015;42:331-6.
9 Foerster S, Unemo M, Hathaway LJ, et al. Time-kill curve analysis and pharmacodynamic modelling for in vitro evaluation of antimicrobials against Neisseria gonorrhoeae. BMC Microbiol 2016;16:216.

10 Wind CM, Schim van der Loeff MF, Unemo M, et al. Test of cure for anogenital gonorrhoea using modern RNA-based and DNA-based nucleic acid amplification tests: a prospective cohort study. Clin Infect Dis 2016;62:1348-55. 